\title{
Local intermittency measure analysis of neutron monitor data
}

\author{
Alexander MacKinnon ${ }^{(1)}$, Sam Rennie ${ }^{2}$ \\ Correspondence \\ 'School of Physics and Astronomy, University of Glasgow, UK, alexander.mackinnon@glasgow.ac.uk \\ ${ }^{2}$ Department of Physics and Mathematics, Nottingham Trent University, UK; School of Physics and \\ Astronomy, University of Glasgow, UK, sam.rennie99@googlemail.com
}

\section{OPEN ACCESS}

This work is published under the Creative Commons Attribution 4.0 International licence (CC BY 4.0) Please note that individual, appropriately marked parts of the work may be excluded from the licence mentioned or may be subject mercopyright conditions other copyright conditions. If such thirdparty material is not under the Creative Commons license, any copying, editing or public reproduction is only permitted with the prior consent of the respective copyright owner or on the basis of relevant legal authorization regulations.

\section{Keywords}

wavelets; time series; neutron monitors

\begin{abstract}
Local Intermittency Measure (LIM) is a development of wavelet analysis particularly suited to the diagnosis of isolated, intermittent events in time series. We construct LIM scalograms of Neutron Monitor (NM) data for an example each of a large GLE and a Forbush decrease. Both kinds of event show distinctive LIM signatures. In the case of the Forbush decrease the method also identifies a second, much smaller event that took place in the same time period. LIM may thus be a useful tool for automated or semi-automated detection of such events in NM data.
\end{abstract}

\section{Introduction}

The data provided by the global neutron monitor (NM) network offer a detailed look at many aspects of solar high-energy phenomena and solar-terrestrial interactions. Semi-regular changes in count rates reflect known periodicities in solar phenomena: the solar rotation rate, the activity cycle. Fourier and wavelet decompositions of the NM count rate time series have been used to extract information about such quasi-periodic behaviour (e.g. Kudela et al. 2010). Some of the most interesting features of NM data, however, are exceptional, one-off events such as ground level enhancements (GLE) and Forbush decreases (FD). Time series analysis methods focusing on quasi-periodic behaviour may not be the best tools for studying them. Here we suggest that Local Intermittency Measure (LIM) may be a useful tool for identifying and characterising such events in NM data. LIM is an application of wavelet analysis first introduced in the study of turbulence in fluids (Farge et al. 1990; Farge, 1992). It has been applied to solar wind data (Bruno et al. 1999), to AE-index data (Consolini \& Chang 1994) and to solar flare X-ray (Dinkelaker \& MacKinnon 2013a, b) and microwave (Gimenez de Castro et al. 2016) data. Here we give a preliminary exploration of its utility for characterising intermittent events in NM data. In the next section we give more details of LIM formalism and what it says about a time series before applying it to NM data to show that it can be effective at identifying GLEs and FDs. Section 4 offers some concluding comments. 


\section{LIM introduced and applied to NM data}

\subsection{Wavelets and LIM}

Let $X_{j}$ be a series of measurements at equally spaced times $\mathrm{j}=1 \ldots \mathrm{N}$. Assuming a wavelet basis function $\psi(t)$ the time $(t)$ and scale $(\tau)$ dependent amplitudes $\mathrm{A}(t, \tau)$ are constructed (e.g. Torrence \& Compo 1998) as

$$
A(t, \tau)=\sum_{j=1}^{N} \psi^{*}\left(\frac{(j-t)}{\tau}\right) X_{j}
$$

We calculate the mean (time-averaged) power at each scale:

$$
W(\tau)=\frac{1}{N} \sum_{j=1}^{N}|A(j, \tau)|^{2}
$$

and define the $\operatorname{LIM} \Lambda(t, \tau)$ as

$$
\Lambda(t, \tau)=\frac{|A(t, \tau)|^{2}}{W(\tau)}
$$

Thus LIM renormalises the wavelet amplitudes in such a way that $\Lambda(t, \tau)>1$ picks out times at which fluctuations on some scale t are over-represented, compared to the average behaviour of the time series. The square of the LIM gives an estimator for the contribution to the kurtosis $K_{4}$ of the distribution of time series increments from fluctuations on scale t (Meneveau 1991). $K_{4}=3$ for a gaussian distribution so the requirement $\Lambda^{2}(t, \tau)>3$ identifies times and scales at which the time series looks inconsistent with a gaussian random walk. See Bruno et al (1999), Dinkelaker and MacKinnon (2013a), and MacKinnon and Rennie (in preparation) for more on interpretation.

Results below are obtained using the Morlet wavelet function which we adopted as standard for this work after some experimentation. We find that it offers a reasonable compromise between accuracy in time-space and in scale-space which is necessary for estimating both the onset times and durations of intermittent events. 


\subsection{GLE of 6 November 1997}

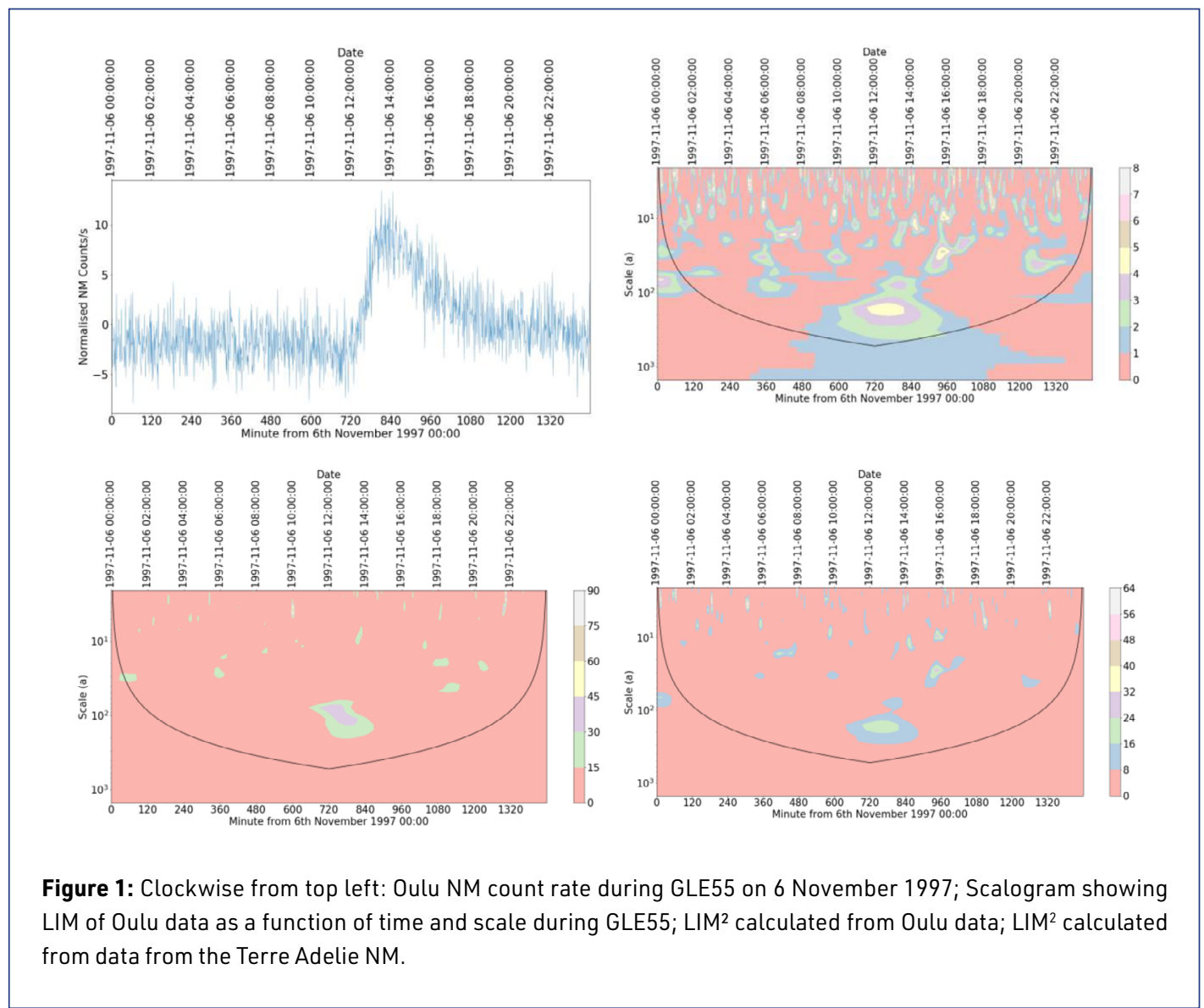

Here we investigate the LIM signatures of a couple of large transient events, firstly GLE55 which took place on 6 November 1997. Figure 1 shows data for this event from the Oulu NM together with scalograms showing the time and scale behaviour of LIM and LIM² during this event. The scalograms show only values satisfying $\Lambda>1$ or $\Lambda^{2}>3$ as discussed above. The occurrence of the GLE is clearly seen as an episode of large LIM values centred on $\sim 1230$ on 6/11/97, at scales of about 200 min which corresponds well with the sudden observed spike in and consequent initial recovery of the secondary cosmic ray flux. Random fluctuations naturally result in many briefer episodes with smaller amplitudes. Most of these are eliminated with the more stringent $\Lambda^{2}>3$ criterion. The LIM ${ }^{2}$ signature of the GLE is also seen in a scalogram calculated using data from the Terre Adelie NM. We can be confident in the reality of this feature since it is present in scalograms constructed using data from two different NM. 


\subsection{Forbush decreases: 14 - 18 February 2011}

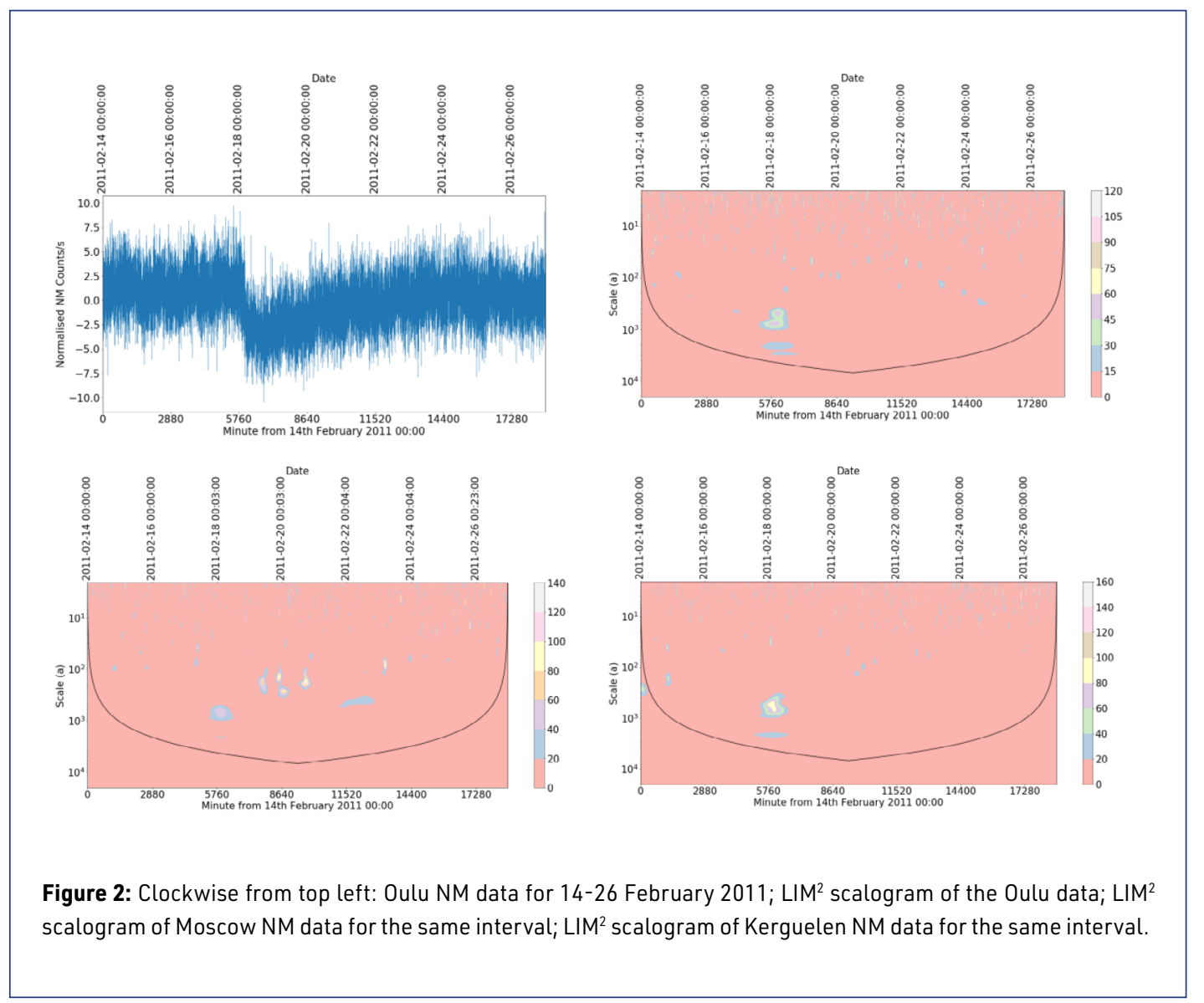

Figure 2 shows data from the Oulu NM for the period 14 - 26 February 2011, clearly showing the large Forbush decrease that took place on 18 February (Lingri et al. 2016). We show LIM² scalograms calculated using these data as well as data from the Kerguelen and Moscow NMs. All three scalograms show a well-defined peak in LIM at the time of the Forbush decrease, at a scale of about 800-900 min which seems to correspond well with the initial, sudden observed decrease in the neutron monitor count rate. All three also show many other features but these are mostly unique to a single NM and thus attributed to data noise. However the scalograms from the Oulu and Kerguelen NM's both show a local maximum on a scale of $100 \mathrm{~min}$, at about 0000 on 15 February. There are maxima in the Moscow data at similar scales at about the same time and similar features are seen in scalograms from several other NMs (Rennie, 2019). These features coincide with the occurrence of a small FD, identified in the Izmiran online catalogue (http://spaceweather.izmiran.ru/eng/ dbs.html, last accessed April 8, 2021) and attributed to a coronal hole. We conclude that the LIM technique has identified the occurrence of this FD, even though it is barely evident to the eye in the high resolution Oulu data shown in figure 2.

\section{Discussion and conclusions}

LIM takes the established method of wavelet analysis and asks when the distribution of increments becomes statistically inconsistent with the previous, average behaviour. In this sense it is similar to the automated FD method described by Light et al. (2020), while also yielding information on the dominant scales in identified events. 
The GLE and FD studied here were selected as random examples. We found that, while local maxima of LIM arise, as expected, from data noise, the events studied here gave rise to well-defined signatures in data from more than one NM. The LIM² scalograms also picked out a much weaker FD, in addition to the large event deliberately selected for study. We conclude that LIM appears to be a promising technique for automated detection of transient events in NM data. We are now testing its performance on a larger sample of data and exploring further its potential for yielding novel diagnostics of space weather events. Results of this fuller study will be reported elsewhere in due course.

\section{Acknowledgments}

We thank the NMDB project (https://www.nmdb.eu/) and the PIs of the Moscow, Terre Adelie, Oulu and Kerguelen NM for providing the data and enabling access. Computer code to construct and display LIM was developed from the wavelet software provided by C. Torrence and G. Compo, available at URL: http://atoc.colorado.edu/research/wavelets/.

\section{References}

Bruno, R., Bavassano, B., Pietropaolo, E., Carbone, V., Veltri, P., 1999, Geophys. Res. Letts. 26, 3158

Consolini, G., Chang, T., 2002, JASTP 64, 541

Dinkelaker, A., MacKinnon, A. L., 2013a, Sol. Phys. 282, 471

Dinkelaker, A., MacKinnon, A. L., 2013b, Sol. Phys. 282, 483

Farge, M., 1992, Ann. Rev. Fluid Mech. 24, 395

Farge, M., Holschneider, M., Colonna, J. F., 1990, Moffatt, H. K. (ed.), Topological Fluid Mechanics, Cambridge University Press

Gimenez de Castro, G., Simões, P. J. A., Raulin, J.-P., Guimarães, O. M., 2016, Sol. Phys. 291, 2003

Kudela, K., Mavromichalaki, H., Papaioannou, A., Gerontidou, M., 2010, Sol. Phys. 266, 173

Light, C., Bindi, V., Consolandi, C., Corti, C., Freeman, C., Kuhlman, A., Palermo, M., Wang, S., 2020, ApJ 896, 133

Lingri, D. Mavromichalaki, H., Belov, A., Eroshenko, E., Yanke, V., Abunin, A., Abunina, M., 2016, Sol. Phys. 291, 1025

Meneveau, C., 1991 J Fluid Mech. 232, 469

Rennie, S, 2019, MSc project report, University of Glasgow

Torrence, C., Compo G. P., 1998, Bull. Am. Met. Soc. 79, 61 